\title{
Hypertrophic Cardiomyopathy at 50
}

\author{
Magdi Yacoub • Iacopo Olivotto $\cdot$ Franco Cecchi
}

Received: 10 November 2009/Accepted: 10 November 2009 / Published online: 4 December 2009

(C) Springer Science+Business Media, LLC 2009

Last year marked the 50th anniversary of the description of $\mathrm{HCM}$ as a disease entity. The ensuing 50 years have witnessed an unprecedented process of discovery related to different aspects of the disease. This process continues today with an ever accelerating rate and utilises modern investigative tools in a timely translational fashion. Apart from addressing important issues in $\mathrm{HCM}$, this process serves as a paradigm for cardiovascular translational research.

This issue of the journal is devoted to outlining in depth several aspects of the disease by experts in the field who have witnessed and contributed to many of these advances. The article by Eugene Braunwald presents a lucid, authoritative, historical account of the "early days". This brings to life many features of the disease while recounting arguments and counter-arguments which surrounds this discovery. An important feature of the disease is the fact that it remains largely undiagnosed in the community with sudden death or advanced stage of the disease being the presenting manifestation. This necessitates the "search" for the disease in the community to determine the true incidence and progression, which is essential for formulating strategies for effective treatment by specialised centres. This important topic is reviewed by Nistri and his colleagues. As mentioned above, sudden cardiac death is one of the most devastating manifestations of the disease, particularly, when it affects young competitive athletes. The possible mechanisms, risk factors and methods of treatment are presented by Barry Maron, drawing on his

M. Yacoub $(\bowtie) \cdot$ I. Olivotto $\cdot$ F. Cecchi

Heart Science Centre, Imperial College London,

Harefield,

Middlesex UB9 6JH, UK

e-mail: m.yacoub@imperial.ac.uk vast experience with HCM for many years. The clinical manifestations and modes of progression are heterogeneous, in spite of the fact that the genetic defect responsible usually affects genes encoding sarcomeric proteins. The particular clinical features "many faces" of HCM are described in detail by Olivotto and colleagues. In addition, the authors provide a unifying hypothesis linking all the manifestations based on developmental origin. From the above accounts, it follows that early definitive diagnosis, preferably in the "preclinical stage" is an important target, as outlined by Caroline Ho. This issue is expanded on by Valentin Fuster and Martin Maron who describe in detail evolving imaging techniques. The issue of exercise in HCM is reviewed by Sharlene Day.

An important aspect of the disease, which influences clinical outcome, is vascular dysfunction at more than one level; this is reviewed by Franco Cecchi and colleagues.

Left ventricular outflow obstruction (dysfunction) is one of the defining features of HCM that is more common than previously thought and have an adverse effect on quality of life as well as survival. Currently, there is no agreement about the exact mechanisms responsible on the type of treatment. As outlined by Yacoub and colleagues, this can only be resolved by defining the structural and functional changes present, which allows evolution and application of tailored techniques for their relief.

The genetic origin of the disease is now well established. This fact, coupled with the observed anxiety of the patients and their relatives, outlines the importance of counselling as an important component of the management scheme. Three articles are included in this issue of the journal describing counselling in adults by Skrzynia, children by Demo et al., as well as a physician's perspective by Baxter.

In spite of the massive amount of knowledge which has accumulated in the last 50 years, the basic mechanisms 
linking the genetic abnormality to the particular phenotype remain unknown. This is an extremely pressing matter as it provides the only means of discovery of a "curative" treatment. The articles by Sivaramakrishnan and colleagues and Ferrantini et al. outlines avenues for molecular and cellular studies to solve this problem. In addition, Semsarian and colleagues describe the use of targeted animal models to contribute to the search.

Finally, Ali Marian and colleagues present some innovative approaches to therapy. It is hoped that this issue of the journal will help in understanding the disease, evolving new strategies and most importantly, stimulate further research. 